\title{
AVALIAÇÃO DE MARCADORES SOROLÓGICOS TREPONÊMICOS E NÃO-TREPONÊMICOS EM DOADORES INAPTOS PARA SÍFILIS ATENDIDOS EM UM HEMOCENTRO BRASILEIRO
}

\section{EVALUATION OF TREPONEMAL AND NON-TREPONEMAL SEROLOGICAL MARKERS IN UNFIT BLOOD DONORS FOR SYPHILIS IN A BRAZILIAN BLOOD CENTER}

Clin Biomed Res. 2019;39(4):284-291

1 Programa de Residência Multiprofissional em Hematologia e Hemoterapia, Centro de Ciências Biológicas e da Saúde, Universidade do Estado do Pará (UEPA). Belém, PA, Brasil.

2 Núcleo de Ensino e Pesquisa (Nepes), Fundação Centro de Hemoterapia e Hematologia do Pará (Hemopa). Belém, PA, Brasil.

Autor correspondente: João Marcos de Oliveira Macêdo joaomaarcos_@live.com Núcleo de Ensino e Pesquisa (Nepes), Fundação Centro de Hemoterapia e Hematologia do Pará (Hemopa)

Travessa Padre Eutíquio, 2109. 66033-230, Belém, PA, Brasil.

\section{João Marcos de Oliveira Macêdo ${ }^{1}$, Camila Fonseca Barroso ${ }^{1}$, Line Alves Monteiro ${ }^{1}$, Laiane Nazaré Silva Nascimento1, Letícia Caroline da Cruz Paula ${ }^{1}$, Renata Bezerra Hermes de Castro ${ }^{2}$ \\ RESUMO}

Introdução: A sífilis é uma doença infecciosa sistêmica de evolução crônica, causada pela bactéria Treponema pallidum, que pode ser transmitida por transfusões de sangue. A avaliação dos marcadores sorológicos na triagem para sífilis em doadores de sangue é fundamental para confirmar se o sangue doado pode estar contaminado com T. pallidum ou se há descarte por reações inespecíficas.

Métodos: A população foi composta por doadores de sangue atendidos em todas as unidades do Hemopa, que atenderam aos critérios do estudo. Houve análise dos resultados obtidos através do VDRL e ELISA e informações socioeconômicas sobre a população: sexo, idade, escolaridade e estado civil, obtidos a partir do registro de doadores.

Resultados: Durante o período do estudo, houve 103.187 doações de sangue. Um total de 883 doadores foram considerados inaptos para sífilis no teste de triagem $(0,86 \%)$ e 271 pessoas $(30,69 \%)$ compareceram ao hemocentro para realização de testes confirmatórios: 50,6\% homens; 49,4\% mulheres; idade média de 34 anos. Mais da metade declarou ter ensino médio completo e a maioria $(75,6 \%)$ relatou ser solteiros. Mais de $50 \%$ dos doadores apresentaram titulação menor que $1 / 16 ; 91,5 \%$ também apresentou reatividade em ELISA. 15 pessoas $(5,5 \%)$ apresentaram outro resultado reagente além da sífilis.

Conclusão: Houve mais de $90 \%$ de resultados concordantes entre teste treponêmico e não treponêmico em nossa triagem laboratorial. Esses resultados podem auxiliar instituições na estratégia de triagem para sífilis em doadores de sangue.

Palavras-chave: Sífilis; doadores de sangue; testes laboratoriais; segurança do sangue; hemoterapia

\section{ABSTRACT}

Introduction: Syphilis is a chronic systemic infectious disease caused by Treponema pallidum, which can be transmitted by blood transfusions. The evaluation of serological markers for syphilis screening in blood donors is crucial to confirm whether the donated blood may be contaminated with $\mathrm{T}$. pallidum or if it is discarded by nonspecific reactions. The aim of this study was to describe results obtained between nontreponemal and treponemal serological tests and sociodemographic profile of blood donors in a Brazilian blood center. 
Methods: The population consisted of all blood donors attended at Fundação Hemopa, who met the study criteria. The results obtained through the VDRL and ELISA tests, as well as socioeconomic information about the population - gender, age, education and marital status - were analyzed.

Results: During the study period, there were 103,187 blood donations. 883 donors were considered unfit for syphilis in the screening test $(0.86 \%)$ and 271 of these (30.69\%) attended the blood center for confirmatory testing: $50.6 \%$ men; $49.4 \%$ women; average age 34 years. More than half reported having completed high school and $75.6 \%$ reported being single. More than $50 \%$ of donors had titers less than $1 / 16$; $91.5 \%$ also had ELISA reactivity. Fifteen people (5.5\%) presented another reagent result in serological screening besides syphilis.

Conclusions: There were more than $90 \%$ concordant results between treponemal and non-treponemal testing in our laboratory screening. These results may assist institutions in the screening strategy for syphilis in blood donors.

Keywords: Syphilis; blood donors; laboratory tests; blood supply safety; hemotherapy

A sífilis é uma enfermidade infecciosa sistêmica de evolução crônica que pode acometer múltiplos tecidos e sistemas, causada pela bactéria Treponema pallidum. A doença apresenta distribuição por todo o mundo, com maiores prevalências em países em desenvolvimento ou subdesenvolvidos, sendo os dados existentes indicadores de não predileção por raça ou sexo, mas evidencia algo recorrente entre os jovens ${ }^{1}$.

Essa bactéria é destruída após alguns minutos de exposição ao ressecamento, baixas temperaturas ou ar, perdendo a sua viabilidade após aproximadamente 72 horas de armazenamento do sangue coletado em temperaturas de refrigeração. A sífilis pode ser transmitida principalmente por meio do contato sexual, por transmissão vertical (congênita) ou por transfusões de sangue, embora a transmissão dessa forma seja rara: o último caso reportado de transmissão de sífilis por transfusão nos Estados Unidos, por exemplo, ocorreu em $1966^{2}$. A triagem para sífilis pelos bancos de sangue e o pouco tempo de sobrevivência da bactéria fora do organismo humano são pontos positivos para evitar a disseminação da doença, por meio de transfusões, na população ${ }^{3,4}$.

O diagnóstico da doença baseia-se por meio de testes sorológicos treponêmicos e não-treponêmicos, uma vez que a bactéria $T$. pallidum não cresce em meios de cultura artificiais, o que seria útil para diferenciar a infecção em curso da imunidade proveniente de uma exposição no passado ${ }^{5}$.

Entre os anos 1970 e 1980, com o aparecimento da síndrome da imunodeficiência adquirida (AIDS), houve um redimensionamento das doenças sexualmente transmissíveis. O papel da sífilis como um fator favorecedor à transmissão do vírus da imunodeficiência humana (HIV) ocasionaria interesse pelo estudo da sífilis e a busca de estratégias para seu controle ${ }^{6}$.
A avaliação dos marcadores laboratoriais para triagem da sífilis é fundamental para confirmar se o sangue doado pode estar contaminado com $T$. pallidum ou se há descarte por reações inespecíficas. A presença de reações inespecíficas implica na perda de doadores saudáveis e em uma redução de componentes sanguíneos em bancos de sangue. $O$ uso de testes de alta sensibilidade na triagem de doadores de sangue é essencial para garantir a segurança transfusional, todavia, é também importante que os testes mantenham alta especificidade para minimizar as possíveis perdas de doadores saudáveis, particularmente em locais com poucos doadores, uma vez que os bancos de sangue brasileiros encontram-se, quase sempre, com níveis de estoque críticos ${ }^{7}$.

\section{OBJETIVOS}

Descrever resultados obtidos entre teste sorológico de triagem (não-treponêmico) e confirmatório (treponêmico) para marcadores imunológicos da bactéria Treponema pallidum na triagem de doadores de sangue na Fundação Hemopa; estimar a soroprevalência de sífilis nas unidades da Hemorrede estadual do Pará; descrever o perfil socioepidemiológico dos doadores de sangue confirmados por testes treponêmicos; e avaliar a ocorrência de resultados verdadeiro e falso-positivos detectados em doadores de sangue considerados inaptos para sífilis.

\section{METÓDOS}

Este projeto foi submetido à Plataforma Brasil, base nacional e unificada de registros de pesquisas envolvendo seres humanos para todo o sistema CEP/Conep, em obediência à resolução $n^{\circ}$ 466/2012 
do Conselho Nacional de Saúde e foi aprovado sob o $n^{\circ}$ CAAE 86098518.4.0000.5174.

O presente estudo trata-se de um estudo retrospectivo, transversal e de caráter observacional. A população estudada foi composta por doadores de sangue atendidos no Hemopa, em todas as unidades da Hemorrede estadual: no Hemocentro Coordenador, em Belém; nos Hemocentros Regionais em Castanhal, Marabá e Santarém; e nos Hemonúcleos de Abaetetuba, Altamira, Capanema, Redenção e Tucuruí, que atenderam aos critérios de elegibilidade na triagem clínica e apresentaram inaptidão no teste laboratorial de triagem para sífilis.

A seleção das amostras dos doadores foi realizada durante os meses de outubro de 2017 a outubro de 2018. Foram critérios de inclusão no presente estudo: doadores inaptos para sífilis na triagem laboratorial, cuja doação e amostra para teste confirmatório tenha ocorrido no período mencionado. Foram critérios de exclusão: doadores inaptos com resultado não reagente para VDRL na segunda amostra, e doadores com volume amostral insuficiente para realização dos testes complementares.

A coleta de dados necessários ocorreu por meio do sistema do banco de sangue, onde as informações socioeconômicas sobre a população está disponível, mediante registro das informações do doador ao se candidatar à doação de sangue. As variáveis obtidas necessárias ao trabalho foram: sexo, idade, escolaridade e estado civil, e foram localizadas a partir do número da amostra ou número da pessoa física (PF), não sendo utilizado em nenhum momento o nome dos doadores. Os números das amostras, resultados observados nos testes realizados e dados sociodemográficos obtidos foram registrados em uma planilha, utilizando o programa Microsoft Excel, para posterior análise descritiva.

Quanto às amostras selecionadas, foram resgatadas amostras de doadores inaptos para sífilis, onde o VDRL apresenta-se reagente após a rotina de triagem laboratorial. Foram resgatadas tanto as amostras de doação, quanto às segundas amostras dos doadores convocados para coleta de $2^{\mathrm{a}}$ amostra (necessária para a realização do teste confirmatório).
Para as amostras do estudo, foram realizados testes treponêmicos e não-treponêmicos. Tais testes foram realizados na primeira e segunda amostra dos doadores que retornaram. Além do VDRL, houve a pesquisa de anticorpos treponêmicos (IgG e $\operatorname{lgM}$ ) através do teste sorológico ensaio imunoenzimático ELISA (ICE Syphilis Murex) (DiaSorin, Branch, Reino Unido).

\section{RESULTADOS E DISCUSSÃO}

No período compreendido de 01 de Outubro de 2017 a 31 de Outubro de 2018, a hemorrede paraense, que é integralmente compreendida pela Fundação Hemopa, recebeu um total de 103.187 doações de sangue, média de 8.598 doações mensais, somando todas as unidades da hemorrede estadual. Durante esse período, 883 doadores foram considerados inaptos para sífilis no teste de triagem e 271 pessoas $(30,69 \%)$ compareceram ao hemocentro após convocação, para realização de testes confirmatórios. Considerando que todos os 883 indivíduos inaptos na triagem sorológica realmente tivessem história prévia de infecção ou uma infecção em curso, observou-se um percentual de inaptidão de $0,86 \%$ de sífilis na população. Esse percentual representa exatamente a quantidade de hemocomponentes descartados devido à reatividade no teste de triagem para sífilis.

Ao analisar individualmente cada unidade da hemorrede, percebe-se que o Hemocentro Regional de Santarém apresenta a maior taxa de inaptidão $(1,08 \%)$, seguido do Hemocentro Regional de Marabá $(1,07 \%)$ e Hemonúcleo de Altamira (1,05\%). O Hemocentro Regional de Castanhal, por outro lado, apresentou a menor taxa de inaptidão por sífilis $(0,63 \%)$, taxa menor, inclusive, que a do Hemocentro Coordenador, em Belém $(0,83 \%)$. A Tabela 1 mostra o percentual de inaptidão para sífilis de todas as unidades da hemorrede paraense, bem como o quantitativo de doações no período do estudo. Observa-se também que o Hemocentro Coordenador foi responsável por pouco mais de $60 \%$ de todas as doações de sangue feitas em hemocentros da hemorrede estadual.

Tabela 1: Inaptidão para sífilis por hemocentro da hemorrede paraense no período de 01/01/2017 a 31/10/2018.

\begin{tabular}{lcccc}
\hline Hemocentro & $\mathbf{N}^{\circ}$ de doações & Qtde. reagente & \% inaptidão & \% doações \\
\hline Abaetetuba & 4.101 & 36 & 0,88 & 3,97 \\
Altamira & 3.238 & 34 & 1,05 & 3,14 \\
Belém & 62.005 & 515 & 0,83 & 60,09 \\
Capanema & 3.048 & 21 & 0,69 & 2,95 \\
Castanhal & 7.518 & 47 & 0,63 & 7,29 \\
Marabá & 7.918 & 85 & 1,07 & 7,67 \\
Redenção & 3.723 & 30 & 0,81 & 3,61 \\
\hline
\end{tabular}


Tabela 1: Continuação.

\begin{tabular}{lcccc}
\hline Hemocentro & $\mathbf{N}^{0}$ de doações & Qtde. reagente & \% inaptidão & \% doações \\
\hline Santarém & 8.515 & 92 & 1,08 & 8,25 \\
Tucuruí & 3.121 & 23 & 0,74 & 3,02 \\
\hline Total & 103.187 & 883 & 0,86 & 100,00 \\
\hline
\end{tabular}

Algumas pesquisas evidenciam a prevalência de sífilis na população de doadores de sangue realizadas no Brasil, onde nota-se certa diferença entre as regiões: $2,1 \%$ no Paraná, $1,1 \%$ em São Paulo, $0,49 \%$ no Amazonas e de $0,3 \%$ no Rio Grande do Sul| ${ }^{8-11}$.

No entanto, para realizar a avaliação do perfil sorológico dos doadores inaptos, bem como seu perfil sociodemográfico, obedecendo os critérios de inclusão e exclusão, 271 indivíduos representam a população deste estudo - pouco menos de $31 \%$ dos doadores inaptos para sífilis no período estudado-, haja vista que $612(69,3 \%)$ doadores não retornaram para realizar o teste confirmatório. É possível que a aflição e angústia que essa convocação causa em muitos doadores seja o motivo para parte considerável desse número. Muitos, também, só retornam quando há necessidade de doação de reposição para alguém próximo. A partir de dados do sistema de informações do hemocentro, percebe-se que muitos doadores podem levar anos para retornar, quando retornam.

Além de avaliar o perfil de resposta sorológica dos doadores frente aos testes laboratoriais, buscouse analisar o perfil sociodemográfico dos doadores inaptos para sífilis. A Tabela 2 demonstra os dados sociodemográficos agregados por sexo, idade, escolaridade e estado civil da população.

Tabela 2: Perfil sociodemográfico dos doadores inaptos para sífilis.

\begin{tabular}{|c|c|c|}
\hline SEXO & $\mathbf{N}$ & $\%$ \\
\hline Masculino & 137 & 50,6 \\
\hline Feminino & 134 & 49,4 \\
\hline \multicolumn{3}{|l|}{ IDADE } \\
\hline $16-25$ & 81 & 29,9 \\
\hline $26-35$ & 77 & 28,4 \\
\hline $36-45$ & 65 & 24,0 \\
\hline $46-55$ & 34 & 12,5 \\
\hline $56-65$ & 13 & 4,8 \\
\hline 66 ou mais & 1 & 0,4 \\
\hline \multicolumn{3}{|l|}{ ESCOLARIDADE } \\
\hline Ensino Fundamental incompleto & 29 & 10,7 \\
\hline Ensino Fundamental completo & 19 & 7,0 \\
\hline Ensino Médio incompleto & 23 & 8,5 \\
\hline Ensino Médio completo & 148 & 54,6 \\
\hline Ensino Superior & 49 & 18,1 \\
\hline Pós-graduação & 3 & 1,1 \\
\hline \multicolumn{3}{|l|}{ ESTADO CIVIL } \\
\hline Solteiro & 205 & 75,6 \\
\hline Casado & 50 & 18,5 \\
\hline União estável & 9 & 3,3 \\
\hline Divorciado & 5 & 1,9 \\
\hline Viúvo & 2 & 0,7 \\
\hline
\end{tabular}

Quanto ao gênero e idade, observamos que, dos doadores com resultado de VDRL reagente, $50,6 \%$ são do sexo masculino e $49,4 \%$ do sexo feminino. Ao analisar o perfil da população reagente para sífilis, Lorandi ${ }^{12}$ percebeu que não havia diferença entre o gênero dos doadores inaptos em Santa Catarina.
No Distrito Federal, entretanto, essa diferença se apresentou diferença significante: $75 \%$ eram homens e apenas $25 \%$ eram mulheres ${ }^{13}$.

A faixa etária de 16-25 anos apresentou maior prevalência de resultados reagentes $(29,9 \%)$. A idade média, calculada a partir da soma da idade de 
Macêdo et al.

todas as 271 pessoas, é 34 anos, sendo 17 a idade mínima e 66 a máxima. Observou-se também nesta população, um decréscimo da porcentagem de reatividade conforme o aumento das faixas etárias, podendo ter relação com a mudança no comportamento sexual na medida que a idade avança. Um estudo afirma que o maior acometimento de sífilis em jovens, especialmente entre 15 e 25 anos, deve-se à maior recorrência de atividade sexual ${ }^{14}$. Alguns estudos, no entanto, mostram um perfil oposto: na medida que a idade aumenta, houve um aumento linear na proporção de doadores soropositivos para sífilis ${ }^{12}$. No estudo de Boff et al. ${ }^{8}$, a faixa etária com maior prevalência de reatividade era entre doadores de 31 e 40 anos (26,7\%).

Em nossa população, 148 pessoas $(54,6 \%)$ declararam ter ensino médio completo; $49(18,1 \%)$ declararam ter diplomação de ensino superior; e 48 $(17,7 \%)$ possuíam, no máximo, nível fundamental. Sandes ${ }^{7}$ encontrou uma associação entre nível de escolaridade e resultados positivos: a chance de uma pessoa que não concluiu o ensino fundamental ter inaptidão sorológica para sífilis foi de 6,59 vezes maior que a chance de pessoas com nível superior; para as pessoas com ensino médio completo, essa chance caiu para 3,37 .

Aproximadamente $70 \%$ da população estudada por Ferreira ${ }^{15}$ não tinha ensino médio completo. Ao somarmos todos os valores da população analisada no presente estudo, com escolaridade inferior a Ensino Médio completo, teríamos um índice menor que $30 \%(26,2 \%)$, discordando dos achados de Ferreira. É muito comum relacionar as infecções sexualmente transmissíveis (IST) às pessoas menos escolarizadas e com menor acesso à informação. No entanto, ressalta-se também a negligência, por parte da população, às ISTs, bem como o uso de métodos para prevenção delas ${ }^{16}$.

Segundo Pirotta e Schor ${ }^{17}$, nem sempre a não-adesão do preservativo deve ser relacionada diretamente com a falta de informação, citando fatores que podem influenciar o não-uso de métodos anticoncepcionais, como esporadicidade e a falta de planejamento das relações sexuais, bem como mitos ligados à relação sexual.

Referente ao estado civil da população estudada, a maioria $(75,6 \%)$ declarou ser solteira, divergindo de outros achados da literatura, cuja proporção entre solteiros e casados eram bem próximas uma da outra, tal como mostram os estudos de Ferreira ${ }^{15}$ e Miranda ${ }^{13}$. Esta diferença pode ser associado à diferença do perfil da população doadora de sangue do estado do Pará. Ferreira ${ }^{15}$ ainda concluiu que doadores com marcadores sorológicos reagentes entre eles, os da sífilis - eram, em maior parte, homens, casados, com menos de 40 anos e baixa escolaridade. E embora Magalhães e colaboradores ${ }^{18}$ evidenciem proporção similar entre homens e mulheres, a maioria das inaptidões sorológicas ocorreram em pessoas com 30 anos ou mais.

Em 2017, foram registrados 119.800 casos de sífilis adquirida no Brasil. Desse número, observou-se que a maior parte das notificações eram de indivíduos com faixa etária de 20 a 29 anos (35,3\%) e 30 a 39 anos $(21,7 \%) ; 18,1 \%$ tinham ensino médio completo e $19,4 \%$ ensino fundamental incompleto. Em $26,1 \%$ dos casos, a informação de escolaridade constava como ignorada. As faixas etárias entre 13 a 19 anos e 20 a 29 anos apresentam uma tendência de aumento no número de casos, quando compara-se o número atual de casos aos de anos anteriores ${ }^{19}$.

Nos países desenvolvidos, relata-se a redução da prevalência de $T$. pallidum, tanto na população em geral quanto entre doadores de sangue. Por outro lado, países emergentes ou subdesenvolvidos apresentam aumento do número de casos de infecção: na Índia, pesquisas sorológicas revelaram altas taxas de soroprevalência, variando entre $9,07 \%$ e 21,9\%; em Gana, a incidência observada foi de 7,5\%; na Tanzânia, 12,7\%; Etiópia, 12,8\%; e na Nigéria, observou-se que $1,1 \%$ dos possíveis doadores de sangue apresentavam infecção por sífilis, contudo um outro estudo relata uma reatividade de $12,4 \%$, 20, 21 .

Após a infecção pelo $T$. pallidum, ocorre um período de incubação entre 10 e 90 dias. Durante esse período, não há sinais da infecção pela bactéria, nem marcadores sorológicos da infecção. Os anticorpos começam a surgir na corrente sanguínea cerca de 7 a 10 dias após o surgimento do cancro duro, sendo detectados inicialmente por testes treponêmicos. Como o T. pallidum não cresce em meio de cultivos artificiais, os testes sorológicos tem grande valor na investigação dessa infecção bacteriana ${ }^{22,23}$. $\mathrm{O}$ uso de testes não treponêmicos na triagem, como neste estudo, pode gerar resultados falso-negativos em situações onde a infecção ainda se encontra nesta fase, possibilitando contaminação por T. pallidum em receptores de sangue.

$\mathrm{Na}$ Fundação Hemopa, todos os testes sorológicos realizados na qualificação de doadores de sangue utilizam o método quimioluminescente para detecção de agentes infecciosos, com exceção da sífilis: para esse marcador, utiliza-se na triagem métodos não-treponêmicos (VDRL). Caso um doador apresente resultados positivos ou inconclusivos para sífilis nesse teste, a bolsa de sangue é bloqueada e descartada, enquanto o doador é notificado para comparecer novamente ao serviço. Ao retornar, o doador é encaminhado ao ambulatório de inaptos da Fundação Hemopa, onde fará uma consulta médica e coleta dos exames confirmatórios para sífilis: se o teste VDRL permanecer reagente na segunda amostra, é realizado o teste treponêmico (ELISA). Caso os resultados se confirmem, o doador receberá esclarecimentos e será encaminhado ao serviço de referência para ter auxílio médico. 
Em relação aos resultados observados no teste de triagem (VDRL) utilizado nos doadores, as titulações variaram entre $1 / 1$ a $1 / 512$. Neste estudo, qualquer evidência de floculação classificou o resultado como reagente. Foi observado que mais de $50 \%$ dos doadores $(57,2 \%)$ apresentaram titulação menor que $1 / 16$, sendo a diluição $1 / 2$ mais frequente nessa faixa. A Tabela 3 apresenta as titulações do teste de triagem e a frequência desses resultados.

Tabela 3: Perfil de resultados obtidos na triagem de doadores.

\begin{tabular}{ccc}
\hline Título & $\mathbf{N}$ & $\mathbf{\%}$ \\
\hline $1 / 1$ & 39 & 14,4 \\
$1 / 2$ & 51 & 18,8 \\
$1 / 4$ & 39 & 14,4 \\
$1 / 8$ & 26 & 9,6 \\
$1 / 16$ & 14 & 5,2 \\
$1 / 32$ & 21 & 7,7 \\
$1 / 64$ & 35 & 12,9 \\
$1 / 128$ & 34 & 12,5 \\
$1 / 256$ & 10 & 3,7 \\
$1 / 512$ & 2 & 0,7 \\
Total & 271 & 100,0 \\
\hline
\end{tabular}

Após realização do teste complementar treponêmico, foi observado que $248(91,5 \%)$ dos doadores inaptos incluídos no presente estudo apresentaram reatividade também no teste treponêmico. Contudo, 23 pessoas $(8,5 \%)$ apresentaram VDRL reagente, porém teste treponêmico ELISA não reagente. Além disso, 15 pessoas $(5,5 \%)$ apresentaram outro resultado reagente, além do VDRL, na triagem laboratorial de doadores, como anti-HCV, anti-HIV e anti-HBc. Este último marcador foi reagente em 11 das 15 amostras (73,3\%).

Segundo Nadal e Framil ${ }^{24}$, o teste VDRL é considerado verdadeiro positivo apenas quando seu título for igual ou maior que $1 / 16$. Corroborando à esta afirmação, foi observado neste estudo que todas as 23 pessoas com resultado não reagente no teste ELISA, apresentaram titulações menores que $1 / 16$ no VDRL, sendo a $1 / 2$ mais frequente $(43,5 \%)$, conforme apresentado na Tabela 4.

É provável que estes resultados não confirmados no teste ELISA e com baixas titulações no teste de triagem provavelmente se deva à falsa positividade do teste VDRL. Sabe-se que algumas situações podem causar esse perfil, mesmo sem infecção pelo T. pallidum, como outras doenças infecciosas (vírus da Hepatite B e C, vírus da Imunodeficiência Humana, hanseníase, leptospirose, malária e mononucleose); doenças autoimunes (lúpus eritematoso sistêmico e demais colagenoses, síndrome antifosfolipídica); hemólise, hepatite crônica, gravidez, idade avançada, vacinações e uso de medicamentos ${ }^{7,24}$. Estudos mostram também que, mesmo em pessoas que nunca tiveram sífilis, podem ser encontradas pequenas quantidades de anticorpos reativos contra antígenos treponêmicos e algumas proteínas do flagelo da T. pallidum ${ }^{7}$.

Tabela 4: Titulação (em VDRL) das amostras não reagentes em ELISA.

\begin{tabular}{ccc}
\hline Título & $\mathbf{N}$ & \% \\
\hline $1 / 1$ & 3 & 13,0 \\
$1 / 2$ & 10 & 43,5 \\
$1 / 4$ & 6 & 26,1 \\
$1 / 8$ & 4 & 17,4 \\
Total & 23 & 100,0 \\
\hline
\end{tabular}

$\mathrm{Na}$ última edição do Boletim de Produção Hemoterápica (HEMOPROD), produzido pela Agência Nacional de Vigilância Sanitária, observa-se que a sorologia reagente para sífilis é a segunda maior causa de descarte de componentes sanguíneos no Brasil. O marcador sorológico Anti-HBc segue consolidado como a principal causa de descartes ao longo dos anos. No Pará, os dados mostram exatamente esse quadro: em 2017, a sorologia reagente para anti-HBc representou $0,76 \%$ dos descartes de hemocomponentes; a sífilis, também segunda maior causa de inaptidão sorológica no Pará, representou $0,64 \%{ }^{25}$.

No entanto, de acordo com os dados divulgados na sexta edição do Hemoprod, a sífilis já é a principal causa de descartes na região sudeste do país, e na região centro-oeste apresenta o mesmo percentual de descartes que o anti-HBc, demonstrando a tendência de redução do percentual de inaptidão pelo anti-HBc nos últimos boletins e aumento em relação à inaptidão por Sífilis ${ }^{26}$. Neste caso, o aumento pode ter relação com a introdução de testes treponêmicos na triagem laboratorial nos bancos de sangue ou mesmo pelo aumento do número de casos da doença no período ${ }^{26}$.

Em um hemocentro do Paraná, testes sorológicos reagentes para Anti-HBc e Sífilis (IgG anti- $T$. pallidum) representaram quase $83 \%$ dos descartes de hemocomponentes por inaptidão sorológica ${ }^{27}$.

Alguns estudos questionam a possibilidade da transmissão de sífilis por meio de transfusões devido aos procedimentos hemoterápicos modernos, uma vez que a bactéria é sensível ao ambiente externo, pode ser destruída por ressecamento e falta de umidade, não resistindo muito tempo fora do corpo humano, e principalmente devido à temperatura: o armazenamento de hemocomponentes abaixo de $20{ }^{\circ} \mathrm{C}$ por mais de 72 horas resulta em danos irreparáveis para a bactéria, perdendo grande parte de sua virulência ${ }^{2,28}$.

Além disso, a transmissão de sífilis por meio de transfusão é considerada rara: o teste para sífilis foi o primeiro a ser obrigatório nos Estados Unidos 
em 1950 e desde então, o último caso reportado de transmissão de sífilis por transfusão nos Estados Unidos ocorreu em 1966,29.

A triagem para sífilis pelos bancos de sangue e o pouco tempo de sobrevivência da bactéria fora do organismo humano, especialmente em baixas temperaturas, como as que se usam para conservar o sangue, são pontos positivos para evitar a disseminação da doença, por meio de transfusões, na população ${ }^{3,4}$. Todavia, é necessária a existência de condutas que visem a prevenção da contaminação, uma vez que este estudo e outros evidenciam a elevada prevalência de sífilis na população doadora de sangue, composta por pessoas hígidas e com baixo comportamento de risco, além de apresentar poucos resultados falso-positivos em nossa triagem.

O aumento do número de notificações de sífilis indica diretamente um aumento da prevalência e incidência da doença na população, culminando na inaptidão temporária dessas pessoas, caso venham se candidatar à doação de sangue: a legislação vigente considerada como inapto à doação, durante 12 meses após a cura, os candidatos à doação que tiveram alguma doença sexualmente transmissível curável, contanto que não haja evidências de infecções repetidas, devido ao maior risco de reinfecção ${ }^{30}$.

Esse aumento das taxas de inaptidão e prevalência da sífilis pode ter relação com a introdução de testes treponêmicos na triagem laboratorial nos bancos de sangue, tornando a pesquisa mais sensível ou mesmo pelo aumento do número de casos da doença no período ${ }^{26}$. Neste estudo, o teste treponêmico foi aplicado somente para confirmações quando houvesse reatividade no teste de triagem. Caso o teste treponêmico fosse aplicado logo na triagem sorológica, o percentual de inaptidão poderia ainda ser maior.

Embora o método quimioluminescente seja, atualmente, a melhor opção para investigação de agentes infecciosos, a maioria dos serviços de hemoterapia brasileiros utilizam para a triagem sorológica testes não treponêmicos pela facilidade, baixo custo do teste e devido à reatividade para sífilis, mesmo após a cura, nos testes treponêmicos ${ }^{7}$, que impossibilitaria muitas pessoas que já tiveram sífilis a doarem sangue. Os serviços de hemoterapia, de acordo com a legislação vigente, podem optar em realizar a triagem para a sífilis com um teste treponêmico ou não-treponêmico, escolhendo o teste que melhor atende a realidade do serviço ${ }^{30}$.

A implementação do método quimioluminescente para sífilis na triagem de doadores incrementaria a segurança transfusional, detectando mais precocemente os anticorpos contra $T$. pallidum. Todavia, pessoas que já tiveram sífilis se tornariam inaptas definitivamente, mesmo curadas, discordando do critério vigente em legislação, que permite a doação 12 meses após a cura. Utilizar o método não-treponêmico é uma alternativa para detectar reaginas em fases mais infecciosas da doença e permitir o uso de hemocomponentes de doadores curados e portadores de cicatriz sorológica para sífilis.

\section{CONCLUSÕES}

A inaptidão sorológica para sífilis na população geral foi de $0,86 \%$; ao analisar cada unidade separadamente, variou de $0,63 \%$ a $1,08 \%$. O percentual representa a quantidade de hemocomponentes descartados devido à inaptidão sorológica.

O perfil socioepidemiológico dos doadores inaptos da hemorrede paraense é composto por homens e mulheres; idade média de 34 anos; a maioria possui ensino médio completo e são pessoas solteiras.

A confirmação da contaminação com T. pallidum é fundamental. Neste estudo, foi observado $91,5 \%$ de verdadeiros positivos, haja vista que 23 pessoas $(8,5 \%)$ apresentaram teste confirmatório negativo, e suas titulações foram menores que $1 / 16$, sendo a $1 / 2$ mais frequente. Reações inespecíficas implicam na perda de doadores saudáveis e impactam na redução de componentes sanguíneos.

Os dados estatísticos gerados pelo trabalho podem auxiliar a instituição na estratégia de triagem para sífilis, bem como a caracterização do perfil dos doadores inaptos da sífilis atendidos na hemorrede estadual do Pará, contribuindo para a avaliação da necessidade de políticas públicas específicas para populações ou grupos específicos.

\section{REFERÊNCIAS}

1. Brasil. Ministério da Saúde. Agência Nacional de Vigilância Sanitária. Manual técnico para investigação da transmissão de doenças pelo sangue. Brasília, DF; 2004. 108 p.

2. Kaur G, Kaur P. Syphilis testing in blood donors: an update. Blood Transfus. 2015;13(2):197-204.
3. Adegoke AO, Akanni OE. Survival of Treponema pallidum in banked blood for prevention of Syphilis transmission. North Am J Med Sci. 2011;3(7):329-32.

4. Baião AM, Kupek E, Petry A. Syphilis seroprevalence estimates of Santa Catarina blood donors in 2010. Rev Soc Bras Med Trop. 2014;47(2):179-85.
5. Seña AC, White BL, Sparling PF. Novel Treponema pallidum serologic tests: a paradigm shift in syphilis screening for the 21st century. Clin Infect Dis. 2010;51(6):700-8.

6. Avelleira JCR, Bottino G. Sífilis: diagnóstico, tratamento e controle. An Bras Dermatol. 2006;81(2):111-26. 
7. Sandes VS. Análise de nova metodologia na triagem sorológica para sífilis em doadores de sangue [dissertação]. Niterói: Universidade Federal Fluminense; 2016.

8. Boff D, Lunkes DS, Kunzler A, Rohr JI. Prevalência de VDRL reagente em doadores do Hemocentro Regional de Cruz Alta - RS, Brasil, no período de 2003 a 2009. Rev Patol Trop. 2011;40(2):179-84.

9. Oliveira VM, Verdasca IC, Monteiro MC Detecção de sífilis por ensaios de ELISA e VDRL em doadores de sangue do Hemonúcleo de Guarapuava, Estado do Paraná. Rev Soc Bras Med Trop. 2008;41(4):428-30.

10. Salles NA, Sabino EC, Barreto CC, Barreto AM, Otani MM, Chamone DF. The discarding of blood units and the prevalence of infectious diseases in donors at the Pro-Blood Foundation/ Blood Center of São Paulo, São Paulo, Brazil. Rev Panam Salud Publica. 2003;13(2-3):111-6.

11. Ferreira CM, Ferreira WA, Motta CL, Vasquez FG, Pinto AF. Reatividade do teste VDRL em bolsas de sangue da Fundação de Hematologia e Hemoterapia do Amazonas-HEMOAM, os custos decorrentes do descarte e estimativa de prevalência de sífilis em doadores de sangue do Estado do Amazonas. DST J Bras Doenças Sex Transm. 2006;18(1):14-7.

12. Lorandi V. Perfil sorológico e sociodemográfico dos doadores do Centro de Hematologia e Hemoterapia de Santa Catarina (HEMOSC): Florianópolis, 2001 a 2006 [trabalho de conclusão de curso]. Florianópolis: Universidade Federal de Santa Catarina; 2009.
13. Miranda LFD. O seguimento de doadores de sangue com sorologia positiva para sífilis na Rede-SUS do Distrito Federal [dissertação]. Brasília, DF: Universidade de Brasília; 2015.

14. Belda Junior W, Shiratsu R, Pinto V. Abordagem nas doenças sexualmente transmissíveis. An Bras Dermatol. 2009;84(2):151-9.

15. Ferreira O. Estudo de doadores de sangue com sorologia reagente para hepatites $B$ e $C$, HIV e sifilis no Hemocentro de Ribeirão Preto [dissertação]. Ribeirão Preto: Universidade de São Paulo; 2007.

16. Ferreira SC, Almeida-Neto $C$, Nishiya AS, Oliveira CD, Ferreira JE Alencar CS, et al. Demographic, risk factors and motivations among blood donors with reactive serologic tests for syphilis in São Paulo, Brazil. Transfus Med. 2014;24(3):169-75.

17. Pirotta KC, Schor N. Reproductive intentions and fertility regulation practices among university students. Rev Saude Publica. 2004;38(4):495-502.

18. Magalhães TA, Teles LF, Nascimento JE, Oliveira LMM Xavier EMS, Aguiar KM, Silva CNM. Prevalence of serological inability of blood donors in regional blood center of Montes Claros, Minas Gerais. Rev Pesqui (Univ Fed Estado Rio J). 2016;8(3):4864-71.

19. Brasil. Secretaria de Vigilância em Saúde. Ministério da Saúde. Boletim Epidemiológico da Sífilis. Brasília, DF; 2018. 43 p.

20. Kumar A, Jyoti V, Prajapati S, Baghel R, Gangane N. Changing trends of syphilis among blood donors in Bastar region, Chhattisgarh: A retrospective study. Community Acquir Infect. 2015;2(2):51-6.
21. Buseri FI, Muhibi MA, Jeremiah ZA. Sero-epidemiology of transfusiontransmissible infectious diseases among blood donors in Osogbo, south-west Nigeria. Blood Transfus. 2009;7(4):293-9.

22. Brasil. Ministério da Saúde. Secretaria de Vigilância em Saúde. Manual Técnico para Diagnóstico da Sífilis. Brasília, DF; 2016. 52 p.

23. Dupin N, Farhi D. Syphilis. La Presse Médicale. 2013;42(4):446-53.

24. Nadal SR, Framil VMS. Interpretação das reações sorológicas para diagnóstico e seguimento pósterapêutico da sífilis. Rev Bras Coloproct. 2007;27(4):479-82.

25. Agência Nacional de Vigilância Sanitária (BR). $6^{\circ}$ Boletim de Produção Hemoterápica. Brasília, DF; 2018.

26. Agência Nacional de Vigilância Sanitária (BR). $4^{\circ}$ Boletim de Produção Hemoterápica. Brasília, DF; 2017.

27. Ramos VF, Ferraz FN. Perfil epidemiológico dos doadores de sangue do Hemonúcleo de Campo Mourão-PR no ano de 2008. SaBios. 2010;5(2):14-21.

28. Vera L, Milka D, Nurith S-L, Eilat S. Prevalence and incidence of syphilis among volunteer blood donors in Israel. J Blood Transfus. 2014;2014:1-7.

29. Katz LM. A test that won't die: the serologic test for syphilis. Transfusion. 2009;49(4):617-9.

30. Brasil, Ministério da Saúde. Portaria de Consolidação $n^{\circ} 5$, de 28 de setembro de 2017. Diário Oficial da União [Internet]. 2017 Set 28 [citado 2019 Dez 4]. Disponível em: https://www.normasbrasil. com.br/norma/portaria-deconsolidacao-5-2017 356384.html

Recebido: 5 mar, 2019 Aceito: 22 out, 2019 